\title{
CDISC SEND Tumor Findings Histopathology Result Category Terminology
}

National Cancer Institute

\section{Source}

National Cancer Institute. CDISC SEND T umor Findings Histopathology Result Category

Terminology. NCI Thesaurus. Code C90004.

The terminology that includes concepts relevant to the Clinical Data Interchange

Standards Consortium (CDISC) Standard for the Exchange of Non-clinical Data (SEND)

tumor findings histopathology result category. 